\title{
Discovering New Polymorphs of Paracetamol via Melt Crystallization
}

Chunhua Tony Hu ${ }^{\mathrm{a}}$, Alexander G. Shtukenberg, ${ }^{\mathrm{a}}$ Melissa Tan, ${ }^{\mathrm{a}}$ Leslie Vogt-Maranto, ${ }^{\mathrm{a}}$ Eric J.

Chan, ${ }^{a}$ Wenqian Xu, ${ }^{\mathrm{b}}$ Jingxiang Yang, ${ }^{\mathrm{a}}$ Mark E. Tuckerman, ${ }^{\mathrm{a}}$ and Bart Kahr ${ }^{\mathrm{a}}$

${ }^{a}$ Department of Chemistry, New York University, 100 Washington Square East, New York, New York 10003 (USA).E-mail: ch98@nyu.edu

${ }^{\mathrm{b}}$ X-ray Science Division, Advanced Photon Source, Argonne National Laboratory, Argonne, Illinois 60439, USA

Paracetamol, aka acetaminophen, is one of the most commonly used medications to treat pain and fever. At the ambient conditions, it exists three crystalline phases, which acts as a model system for studying transformations among phases of molecular crystals. During crystallization from the melt and the glass above $0{ }^{\circ} \mathrm{C}$, three new ambient polymorphs of paracetamol, namely VII, VIII, and IX, were discovered through polarized microscope, Raman spectroscope, and powder diffraction methods. The crystal structure of Form VII was solved using a combination of powder X-ray diffraction and computational techniques. The relative stability of the new phases with respect to the known three phases was examined and growth kinetics was also discussed in this presentation.

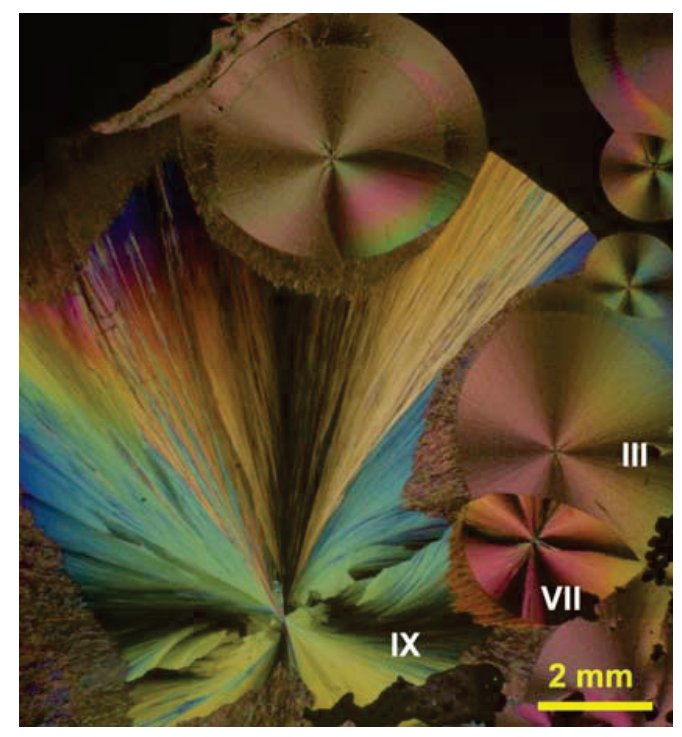

Figure 1. Polarized light micrograph of paracetamol polymorphs between crossed polarizers. Most of the area is occupied by a large spherulite of $\mathbf{I X}$. 